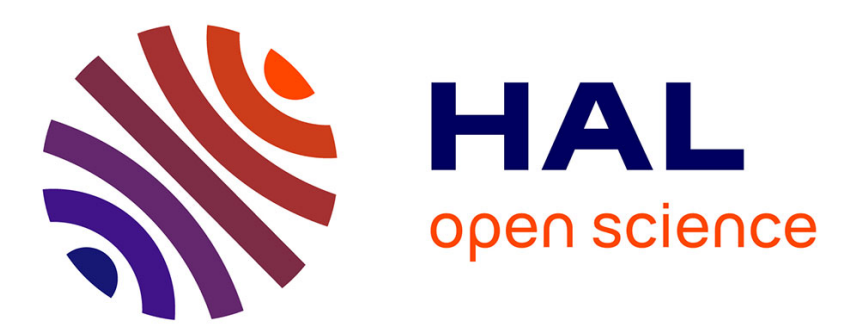

\title{
Assessment of the airborne sound insulation from mobility vibration measurements; a hybrid experimental numerical approach
}

\author{
N.B. Roozen, Quentin Leclere, D. Urbán, T. Méndez Echenagucia, P. Block, \\ M. Rychtáriková, C. Glorieux
}

\section{To cite this version:}

N.B. Roozen, Quentin Leclere, D. Urbán, T. Méndez Echenagucia, P. Block, et al.. Assessment of the airborne sound insulation from mobility vibration measurements; a hybrid experimental numerical approach. Journal of Sound and Vibration, 2018, 432, pp.680 - 698. 10.1016/j.jsv.2018.06.058 . hal-01859993

\section{HAL Id: hal-01859993 \\ https://hal.science/hal-01859993}

Submitted on 11 Jul 2020

HAL is a multi-disciplinary open access archive for the deposit and dissemination of scientific research documents, whether they are published or not. The documents may come from teaching and research institutions in France or abroad, or from public or private research centers.
L'archive ouverte pluridisciplinaire HAL, est destinée au dépôt et à la diffusion de documents scientifiques de niveau recherche, publiés ou non, émanant des établissements d'enseignement et de recherche français ou étrangers, des laboratoires publics ou privés. 


\title{
Assessment of the airborne sound insulation from mobility vibration measurements; a hybrid experimental numerical approach
}

\author{
N.B. Roozen ${ }^{1) *}$, Q. Leclère ${ }^{2)}$, D. Urbán ${ }^{3)}$, T. Méndez Echenagucia ${ }^{4)}$, \\ P. Block ${ }^{4)}$, M. Rychtáriková ${ }^{5)}$, C. Glorieux ${ }^{1)}$ \\ 1) KU Leuven, Laboratory Acoustics, Department of Physics and Astronomy, Leuven, Belgium. \\ 2) Univ Lyon, INSA-Lyon, LVA EA677, F-69621 Villeurbanne, France. \\ 3) Slovak University of Technology in Bratislava, Radlinského 11, 81005 Bratislava, Slovakia. \\ 4) ETH Zurich, Institute of Technology in Architecture, Zurich, Switzerland. \\ 5) KU Leuven, Faculty of Architecture, Gent / Brussel, Belgium.
}

\section{Summary}

A new measurement procedure is proposed to assess the airborne sound insulation of a partition under diffuse sound field excitation using mobility measurements combined with a numerical procedure. The advantage of this hybrid approach is that the diffuse acoustic field does not need to be physically created, thus avoiding problems related to the generation of such fields at low frequencies. Furthermore, the acoustic properties of both the source and receiving rooms will not affect the determination of the sound reduction index $R$. The proposed method is especially suited for frequencies below the so called Schroeder frequency of the room, and is complementary to the standardized measurement approaches as described in ISO 10140-2:2010.

The measurement part of the proposed procedure involves the measurement of the mobility by forcing the partition along a grid of excitation points (e.g. by means of a shaker) and measuring its response along a grid of response points (e.g. by means of a scanning laser Doppler vibrometer). Using the resulting matrix of mobility transfer functions, the vibrational response of the partition excited by a diffuse acoustic field is numerically calculated, from which the radiated sound power is computed using the Rayleigh integral. Thus the reliance on source and receiving rooms used in standard sound insulation testing is removed entirely. The proposed method provides an estimate that only depends on the properties of the partition.

The method was tested in an acoustic laboratory on a single layer glass plate of $1.35 \times 1.54 \mathrm{~m}^{2}$, as well as on a funicular shell structure with dimensions of $3 \times 3 \mathrm{~m}^{2}$. Comparisons with analytical models and standardized ISO10140-2:2010 measurements confirm the validity of the results.

\footnotetext{
* Now at A\&Z Acoustics, Bratislava
}

\section{Introduction}

In contemporary architectural design, the increased implementation of lightweight constructions with lower sound insulation at low frequencies, combined with the more frequent use of hi-fi systems and home cinema with relatively high power at low frequencies, has increased people's awareness of low frequency noise in their living environment. Indeed, adverse effects on the human well-being have been reported[1]. In automotive and aviation industries similar issues arise due to the use of lightweight structures.

The increase in interest in low frequency sound insulation has stimulated research activities on two fronts. On one hand, questions on the importance of the low frequency part of the spectrum in people's subjective perception of sound insulation have been tackled by listening tests [2], aimed at designing single number quantities that include the frequency range below $100 \mathrm{~Hz}[3,4]$. In current standards the assessment of sound insulation in frequency range down to $50 \mathrm{~Hz}$ is still optional [5]. On the other hand, researchers have been giving extra attention $[6,7,8]$ to the problem of high uncertainties on values of the sound reduction index $R$ obtained at low frequencies by airborne sound insulation measurements according to ISO10140-2:2010[9].

Indeed, sound insulation measurements in the lower frequency range are difficult to perform. Due to the reduced density of room acoustic eigenmodes, microphone based measurements are strongly affected by non-uniformity of the sound pressure field at low frequencies $[10,11,12]$, resulting in poor reproducibility between laboratories[13, 3]. Bravo et al. [14] succeeded to reduce the effect of room acoustic issues in the source room by using a number of loudspeakers close to the panel, which were driven independently in order to create a diffuse incident field down to the lowest frequencies of interest. An optimal set of loudspeaker excitation signals was generated by mak- 
ing use of measured transfer functions between locations close to the speakers and locations close to a grid of points along the building element. Bravo et al. measured the radiated power into the receiving room by means of a dense array of acoustic intensity measurements. However, it turned out that this approach was affected by room acoustic effects in the receiving room. The latter effects were avoided in an approach proposed by Roozen et al. [15] in which the vibrational pattern of the partition was measured by means of laser Doppler scanning vibrometry. The radiated sound power was then calculated by means of a Rayleigh integral [16]. Although combining the excitation scheme of Bravo et al. and the detection scheme of Roozen et al., would be a logical next step, the experimental effort to combine the two approaches was found to be very large [17].

Here, a hybrid experimental-numerical approach is proposed in which the sound transmission through the partition is determined without physically creating a diffuse field in the source room. The partition is mechanically excited along a grid of excitation points (at the 'source' side) and the response is measured along a grid of response points (at the 'receiving' side). The diffuse excitation field is described by well-known relationships for the spatial correlation of a diffuse acoustic field to numerically construct a cross spectral matrix for the excitation on the source side. Using the cross spectral matrix, the response of the structure is computed on the basis of the experimentally determined mobility transfer functions. The incident and radiated power, needed to determine the sound reduction index $R$ are then determined from, respectively, the (virtual) exciting sound pressure levels, and the structural response to the diffuse field excitation, computed by means of the Rayleigh integral as in Roozen et al. [15].

The computation and measurement of mobility transfer functions is a classical subject in the field of noise and vibration $[18,19]$. Also the spatial correlation functions of a diffuse acoustic field (see Section 2.1.1) are well-known. However, the use of these spatial correlation functions to numerically compute the vibrational response of the partition from a measured matrix of structural mobility transfer functions, is new, to the knowledge of the authors. In combination with the use of the Rayleigh integral the influence of the room acoustic effects of the source and receiving rooms - used in standard insulation testing - is completely removed from the sound insulation measurement.

In literature, point mobility measurements were also used by Piana [20] to obtain an estimate of the sound reduction index of a single leaf panel by making use of an analytical model. Unlike in that work, in which the finite dimensions of the panel were not taken into account, our approach makes use of measured vibration data along the whole sample, avoiding the use of geometrical approximations, even for complex geometries.

Marchetto et al. [21] proposed a combination of mobility transfer functions measurements and acoustic transfer function measurements to obtain the sound reduction index of a building element. The measurement of the acoustic transfer functions requires the use of monopole and dipole sources. Although this method eliminates the effect of the source room acoustics, due to the airborne detection of the radiated sound, the results are still affected by room acoustic effects of the receiving room, especially for low frequencies.

Chazot and Guyader [22] numerically predict the sound reduction index of finite double panels, taking into account source room dimensions, absorption, excitation position, and panel location. The difference with our approach is that we determine the sound reduction index of the partition for diffuse field excitation from measured mobilities. Furthermore in the approach presented in this paper, the room acoustic effects of the source and receiving rooms are eliminated, thus yielding an estimate of the sound reduction index that only depends on the properties of the partition itself.

The remainder of the paper is organized as follows. Section 2 deals with the theory to numerically construct the cross spectrum of a diffuse sound field, and to use this to compute the spectrum of the structural vibrations of the partition from a matrix of measured mobilities. This section also explains the subsequent steps to compute the radiated sound power from the computed vibration spectrum, and to determine the sound reduction index. Validating experiments that were performed on a single layer glass plate are discussed in section 3 . In section 4 measurements on a funicular shell ceiling system are discussed. The experimental results are compared both with results obtained with a numerical finite element model of the building element and with measurements according to ISO10140-2:2010. Conclusions are drawn in section 5. In the appendix, an analytical model that predicts the airborne sound reduction of a simply supported plate under diffuse acoustic excitation, including radiation damping effects, is discussed. Also a procedure to compute the -Rayleigh integral based- acoustic radiation impedance matrix is presented.

\section{Theory}

\subsection{Determination of the sound reduc- tion index $R$ under diffuse acoustic field excitation}

In the proposed method, a diffuse acoustic field is numerically synthesized, the structural response of the device is determined using the measured matrix of 


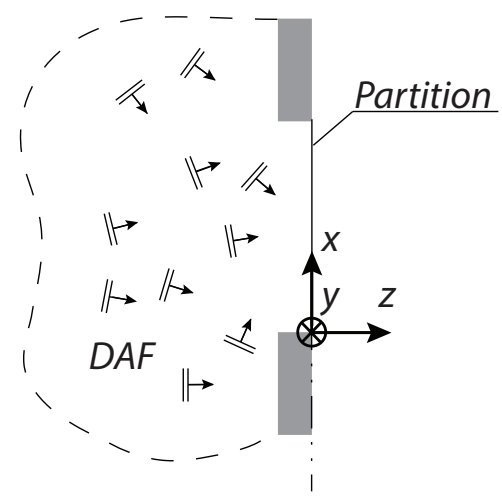

Figure 1: The diffuse acoustic field (DAF), envisaged as an infinite set of uncorrelated plane waves.

mobilities, and the radiated sound power computed by means of the Rayleigh integral. The following subsections describe the theory in more detail.

\subsubsection{The synthesis of an acoustic diffuse field and the determination of the vibra- tional response}

Considering a diffuse acoustic field [21, 23, 24, 25], the cross spectral density of the sound pressure between two positions $x_{i}$ and $x_{j}$ on a rigid wall is given by

$$
G_{i j}\left(x_{i}, x_{j}, \omega\right)=2 \overline{p_{e}^{2}}(\omega) \frac{\sin \left(k\left\|x_{i}-x_{j}\right\|\right)}{k\left\|x_{i}-x_{j}\right\|}
$$

where $k=\omega / c$ is the acoustic wavenumber, $\omega$ is the angular frequency and $c$ is the speed of sound. $\overline{p_{e}^{2}}$ is the averaged quadratic pressure in the diffuse field, the factor 2 arises from the pressure doubling that occurs on a rigid surface. The assumption of rigidity is easily satisfied, as typically the impedance of a wall is orders of magnitude larger than the one of air.

The diffuse field is often mathematically described as a summation of an infinite set of uncorrelated plane waves incident upon the partition with uniformly distributed angles of incidence, as illustrated in Fig. 1. For that reason, many papers revert to the wavenumber domain $\left(\mathbf{k}_{\mathbf{x}}, \mathbf{k}_{\mathbf{y}}\right)$ (e.g. [21]). However, the description of a diffuse field by an infinite set, or in practice by a large set of uncorrelated plane waves, is computationally very expensive. In this paper it is shown that the diffuse field can be described directly in the spatial domain $(\mathbf{x}, \mathbf{y})$, with clear advantages.

In this work, the structure under test is characterized by transfer function measurements between a discrete set of forces $\mathbf{f}(\omega)$ at $N_{e}$ excitation points $\mathbf{x}_{e}$ and a discrete set of velocities $\mathbf{v}(\omega)$ at $N_{r}$ response points $\mathbf{x}_{r}$, such that its response to a distributed load field can be formulated as follows

$$
\mathbf{v}(\omega)=\mathbf{H}(\omega) \mathbf{f}(\omega)
$$

where $\mathbf{H}$ is the $N_{r} \times N_{e}$ matrix of mobility transfer functions. This linear relationship between excita- tions and responses can be formulated using averaged cross-spectra as follows

$$
\mathbf{G}_{\mathbf{V V}}=\mathbf{H ~ G}_{\mathbf{F F}} \mathbf{H}^{*}
$$

where the frequency dependence $\omega$ is omitted for the sake of brevity. In order to synthesize the response of the structure to a diffuse field, the input force cross spectral matrix $\mathbf{G}_{F F}$ (in units $\mathrm{N}^{2}$ ) has to be converted into one for pressure (units $\mathrm{Pa}^{2}$ ) in order to use as an input the cross spectral matrix of a diffuse field. To do that, a portion of the structure's surface has to be associated with each excitation point, and the relation between the force and pressure distribution can be expressed as follows

$$
\mathbf{p}_{e}=\mathbf{d} \mathbf{S}^{-1} \mathbf{f}
$$

where $\mathbf{p}_{e}$ is the $N_{e} \times 1$ vector of excitations in units $\mathrm{Pa}$, and $\mathbf{d} \mathbf{S}$ a diagonal matrix whose entries correspond to the surfaces allocated to input points, verifying $\operatorname{Tr}(\mathbf{d} \mathbf{S})=S$, where $S$ is the total surface of the structure.

The response of the structure to a discretized diffuse field can then be synthesized as

$$
\mathbf{G}_{\mathbf{V V}}=2 \overline{p_{e}^{2}} \mathbf{H} \mathbf{d} \mathbf{S} \mathbf{G}_{\mathbf{D}} \mathbf{d} \mathbf{S} \mathbf{H}^{*}
$$

where $\mathbf{G}_{\mathbf{D}}$ is a $N_{e} \times N_{e}$ matrix whose entries correspond to normalized diffuse field cross spectra (considering $\overline{p_{e}^{2}}=1 / 2$ in Eq. (1)).

\subsubsection{Determination of the radiated sound power}

The acoustic pressure at the receiving side can be determined from the measured vibration velocity field $\mathbf{v}$ of the structure by numerically solving the Rayleigh integral. This step can be expressed in terms of the radiation impedance matrix [16, 26, 27] as follows (see Appendix A for an expression for $\mathbf{Z}$ ):

$$
\mathbf{p}_{\mathbf{r}}=\mathbf{Z v}
$$

where $\mathbf{p}_{\mathbf{r}}$ is a $N_{r} \times 1$ vector of acoustic pressures at the receiving side of the partition, and $\mathbf{Z}$ the $N_{r} \times N_{r}$ radiation impedance matrix. Pressure-velocity cross spectra, that are needed to calculate the radiated power, are obtained with

$$
\mathbf{G}_{\mathbf{P}_{\mathbf{r}} \mathbf{V}}=\mathbf{Z G}_{\mathbf{V V}}
$$

The active sound power $\Pi_{r}$ being radiated by the partition can be computed by

$$
\Pi_{r}=\operatorname{Tr}\left(\mathbf{d} \mathbf{S} \operatorname{Re}\left(\mathbf{G}_{\mathbf{P}_{\mathbf{r}} \mathbf{V}}\right)\right)
$$

\subsubsection{Determination of the incident sound power and sound reduction index $R$}

In a diffuse field, the acoustic power incident upon the structure is equal to [28]

$$
\Pi_{e}=S \frac{\overline{p_{e}^{2}}}{4 \rho c}
$$


where $\overline{p_{e}^{2}}$ is the spatially averaged mean square pressure in the diffuse field.

Finally, the sound reduction index $R$ can be computed from the ratio of incident and radiated power as

$$
\begin{aligned}
R & =10 \log \left(\frac{\Pi_{e}}{\Pi_{r}}\right) \\
& =-10 \log \left(\frac{8 \rho c}{S} \operatorname{Tr}\left(\mathbf{d} \mathbf{S} \operatorname{Re}\left(\mathbf{Z H} \mathbf{d} \mathbf{S} \mathbf{G}_{\mathbf{D}} \mathbf{d} \mathbf{S} \mathbf{H}^{*}\right)\right)\right)
\end{aligned}
$$

where log is the logarithmic base 10. In the particular case of the use of the same regular mesh of $N$ points for excitation and response dofs, such that elements of $\mathbf{d} \mathbf{S}$ are all equal to $S / N$, Equation (10) becomes

$$
R=-10 \log \left(\frac{8 \rho c S^{2}}{N^{3}} \operatorname{Tr}\left(\operatorname{Re}\left(\mathbf{Z H} \mathbf{G}_{\mathbf{D}} \mathbf{H}^{*}\right)\right)\right)
$$

\subsection{Discretization criteria}

The accuracy of the proposed method depends upon the discretization, i.e. the number of excitation points $N_{e}$ and response points $N_{r}$. Discretization takes place at the excitation side (Eq. 4, Eq. 5) and at the radiation side (Eq. 6 and Eq. 8). On both sides, discretization requirements as a function of both structural and acoustic wavelengths have to be met carefully. The wavenumber content of the diffuse field is characterized by a maximum wavenumber equal to $2 \pi / \lambda_{a}$, corresponding to plane waves of grazing incidence. This excitation field acts on the structure, being represented by the matrix of mobility functions. For an accurate representation of the mobility functions the discretization thus has to meet also some spatial sampling criteria with respect to the structural wavelength. On the reception side, the same criteria need to be considered, related to the correct representation of the acoustic radiation mechanism. Finally, a general rule of thumb can be written as follows

$$
\begin{aligned}
& \sqrt{S / N_{e}}<\min \left(\lambda_{a}, \lambda_{s}\right) / n \\
& \sqrt{S / N_{r}}<\min \left(\lambda_{a}, \lambda_{s}\right) / n
\end{aligned}
$$

where $\lambda_{a}$ and $\lambda_{s}$ are acoustic and structural wavelength, respectively, and where $n$ is the number of points per wavelength. Following Shannon's sampling criterion, $n$ should be greater than 2 . In Finite Element modeling, a criterion of $n=6$ points by wavelength is often advised so as to ensure convergence. In the present work, the measurement time has also to be taken into account, the high frequency limit is considered to be reached when $n=3$.

\subsection{Considerations regarding the re- quired experimental effort}

The experimental effort to measure the $N_{r} \times N_{e}$ matrix of transfer functions $\mathbf{H}$ can be significant, especially when for reasons of fine sampling $N_{r}$ and $N_{e}$

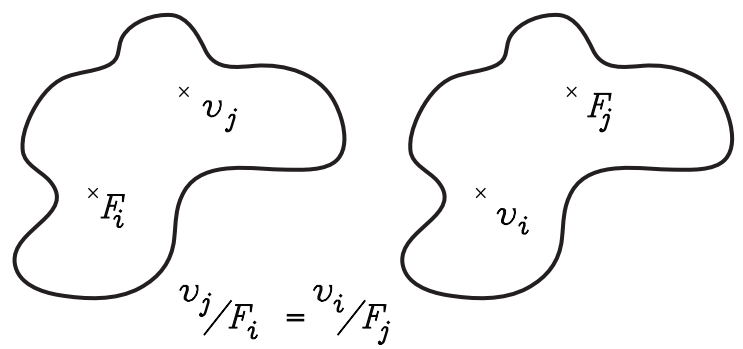

Figure 2: Reciprocity principle.

need to be large. To reduce the experimental effort, the principle of reciprocity can be exploited, provided that the points of excitation coincide with the points where the response is measured. As illustrated in Fig. 2, the transfer function $v_{j} / F_{i}$ as indicated in the left part of the figure is the same as the transfer function $v_{i} / F_{j}$ on the basis of reciprocity of linear time-invariant systems. Using reciprocity reduces the amount of transfer functions that need to be measured from $N^{2}$ (with $N=N_{r}=N_{e}$ ) to $\left(N^{2}-N\right) / 2+N$. Note that thanks to reciprocity only half of the crossmobility points needed to be measured. The number of point-mobility measurements stays the same. Thus, exploiting reciprocity reduces the measurement effort by somewhat less than a factor 2 .

In case the geometry of the partition is symmetric, the number of measurements can be reduced as well. For instance, the funicular shell that will be discussed in section 4, and illustrated in Fig. 7, has 3 axes of symmetry. This type of symmetry reduces the number of measurement points by approximately a factor $2^{3}=8$. Also in this case, the reduction in the measurement effort is somewhat smaller than this theoretical number due to the fact that points on the symmetry line should always be measured. The additional use of reciprocity properties can reduce the required number of measurement even further by approximately a factor 2 .

\section{Assessment of the sound re- duction index for a single layer glass plate}

This section discusses the assessment of the sound reduction index of a single layer glass plate at low frequencies, to validate the mobility-based approach. The results are compared with an analytical model.

\subsection{Measurement set-up}

A single layer glass plate was mounted in the opening of the test suite at the acoustic laboratory of $\mathrm{KU}$ Leuven, in which the opening and the glass plate have dimensions $S=1.35 \times 1.54=2.08 \mathrm{~m}^{2}$. The single layer glass plate had a thickness of $12 \mathrm{~mm}$. 
The glass plate was positioned in the opening by a wooden frame sealed by means of rubber mastic. A matrix of mobility transfer functions $\mathbf{H}$ (see Eq. 2) was measured at an excitation grid of $3 \times 4=12$ points $\left(\sqrt{S / N_{e}}=0.41 \mathrm{~m}\right)$, and a response grid of $7 \times 7=49$ points $\left(\sqrt{S / N_{e}}=0.21 \mathrm{~m}\right)$. The excitation and response grid distances were uniformly distributed over the glass panel. Thus the matrix of mobility transfer functions $\mathbf{H}$ had a dimension of $N_{r} \times N_{e}=12 \times 49$, for each frequency considered. The mobilities were measured in a frequency range from $10 \mathrm{~Hz}$ up to 300 $\mathrm{Hz}$, with a frequency resolution of about $0.1 \mathrm{~Hz}$.

The single layer glass plate was mechanically excited by means of a mini-shaker (see Figure 3). The response of the glass plate was measured by means of a scanning laser Doppler vibrometer. The mini-shaker was equipped with an impedance head (Bruel\&Kjaer type 8001), comprising a force cell to measure the transmitted force.

In this case, symmetry of the partition, nor reciprocity properties were exploited to reduce the measurement effort, as the response measurements were automated through the use of a scanning vibrometer measurement system.

Based on Kirchhoff's thin shell theory [31], the structural wavelength was estimated by means of the formula

$$
k_{f}=\left(\omega^{2} \frac{\rho h}{D}\right)^{1 / 4}
$$

where $\omega$ denotes the exciting angular frequency, $D$ is the dynamic flexural rigidity defined by

$$
D=\frac{E h^{3}}{12\left(1-\nu^{2}\right)}
$$

where $h$ is the plate thickness, $E$ is the Young's modulus and $\nu$ is the Poisson's ratio. Using a Young's modulus $E=56 \mathrm{e} 9 \mathrm{~Pa}$, density $\rho=2400 \mathrm{~kg} / \mathrm{m}^{3}$, Poisson's ratio $\nu=0.24$, thickness $h=12 \mathrm{e}-3 \mathrm{~m}$, the structural wavelength $\lambda_{f}=2 \pi / k_{f}$ is estimated to be about $1 \mathrm{~m}$ at $100 \mathrm{~Hz}$. The acoustic wavelength at $100 \mathrm{~Hz}$ equals 3.4 m. Thus, with $\sqrt{S / N_{e}}=0.41 \mathrm{~m}$ and $\sqrt{S / N_{r}}=0.21 \mathrm{~m}$, the discretization criteria as described in Section 2.2 are fulfilled up to $100 \mathrm{~Hz}$ for this specific case.

\subsection{Results; A comparison of measure- ments and simulations}

Using the theory as described in section 2, the sound reduction index $R$ of the glass plate under a diffuse field excitation, exploiting the measured matrix of mobility transfer functions $\mathbf{H}$, was computed. The results are shown in Fig. 4.

Following the same procedure the sound reduction index $R$ was computed, using a numerically computed matrix of mobility transfer functions $\mathbf{H}$. The

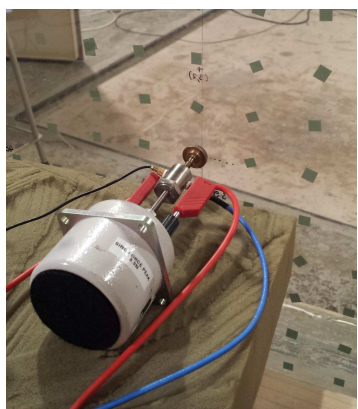

Figure 3: Shaker test set up of the single layer glass plate of size $0.012 \times 1.35 \times 1.54 \mathrm{~m}$.

numerical model comprises a modal model, representing a simply supported plate in vacuum with dimensions $L_{x}=0.35 \mathrm{~m}, L_{y}=0.22 \mathrm{~m}$, Young's modulus $E=56 \mathrm{e} 9 \mathrm{~Pa}$, density $\rho=2400 \mathrm{~kg} / \mathrm{m}^{3}$, Poisson's ratio $\nu=0.24$, thickness $h=12 \mathrm{e}-3 \mathrm{~m}$. The Young's modulus was tuned such to get a reasonable correspondence of the first resonance frequency with the measured resonance frequency of the glass plate. For the numerical modal model a rather fine patch discretization $N_{e}=N_{r}=20 \times 20=400$ was employed. The thus obtained analytical results are presented in Fig. 4 as well.

In experimental circumstances, acoustic radiation damping is naturally included in the measured matrix of mobility transfer functions $\mathbf{H}$. However, when performing analytical or numerical simulations, where an in-vacuo structure is being considered, obviously this damping effect is lacking. Having computed a matrix of transfer functions $\mathbf{H}$ "in-vacuo", the radiation damping can be taken into account afterwards as described in Appendix B. Both the analytical results with and without radiation damping are presented in Fig. 4.

Radiation damping effects are essential in order to obtain physically correct sound reduction indices. The sound reduction index should in all cases be non-negative, as it is physically impossible to radiate more acoustic energy than the incident acoustic energy. Note that in literature some papers show results that violate this physical rule, see for instance [32] (see also Fig. 16 in Appendix B).

The correspondence between the shaker-based sound reduction index and the sound reduction index as predicted by the analytical model is qualitative and quantitatively reasonably good up to a frequency of about $100 \mathrm{~Hz}$ (see end of Section 3.1). At higher frequencies the excitation and response grids become too coarse. At very low frequencies, $<15 \mathrm{~Hz}$, the shaker based sound reduction index becomes noisy, because the physical response of the glass plate is dropping too much, thus decreasing the signal to noise ratio of the laser Doppler signal.

This experiment validates the approach outlined in section 2 . 


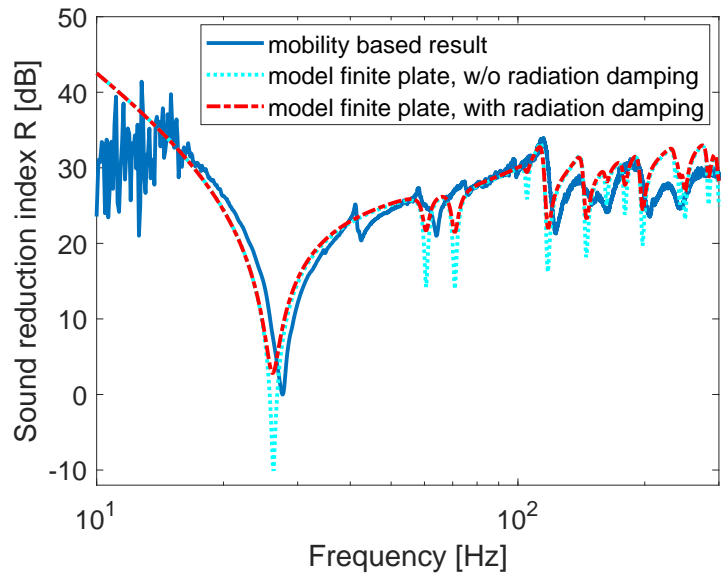

Figure 4: Sound reduction index $R$ of a single layer glass plate of size 0.012 × 1.35 × $1.54 \mathrm{~m}$, mobilitybased result (blue solid curve), analytical model without radiation damping (magenta dotted curve), analytical model with radiation damping (red dashed curve).

\section{Assessment of the sound re- duction index for a funicular shell}

This section discusses the assessment of the sound reduction index of a funicular shell ceiling structure at low frequencies by means of the proposed method. The experimental set-up is discussed in section 4.1, followed by a discussion of the measured operational deflection shapes at resonance frequencies in section 4.2 and the measurement of the sound reduction index from the mobility data in Section 4.4.

A comparison of the mobility-based prediction of the sound reduction index of a funicular shell with the microphone based approach according to the ISO 10140-2:2010 standard is given in Section 4.3. The results are also compared with the results obtained with a numerical numerical finite element method (FEM) model. The model and its results are discussed in Section 4.5. The FEM model is also used to perform a convergence study of the grid discretization used for the funicular shell.

\subsection{Measurement set-up}

The funicular shell ceiling system was cast in the horizontal opening of the test suite at the acoustic laboratory of KU Leuven, having dimensions outer dimensions $3 \times 3=9 \mathrm{~m}^{2}$ (inner dimensions $2.8 \mathrm{~m} \times$ $2.8 \mathrm{~m}$ ). Figures 5 and 6 give a view from above and from below the funicular shell, respectively.

The matrix of mobility transfer functions $\mathbf{H}$ (Eq. 2) was measured by exciting the funicular shell from above (the rib-side) by means of a shaker (see Fig.
$5)$. Both excitation points on the ribs and excitation points in the bays between the ribs were used. The response of the funicular was measured from below (see Fig. 6) by means of a scanning laser Doppler vibrometer measurement system. Pieces of retro-reflecting tape were glued on the dome of the funicular shell to increase the optical reflection at the measurement points. The shaker was equipped with a stinger and an impedance head (Bruel\&Kjaer type 8001), the latter comprising a force cell to measure the transmitted force.

To reduce the measurement effort, the symmetry of the funicular shell structure was exploited. As illustrated in Fig. 7, the funicular shell structure is symmetric with respect to the horizontal axis, the vertical axis and with respect to the diagonal. Exploitation of this symmetry reduced the number of measurement points by approximately a factor of 8 . Reciprocity properties to reduce the measurement effort were not used.

The response of the dome of the funicular shell were measured along a full grid of $15 \times 15=225$ points, by means of a scanning laser Doppler measurement system (Polytec PSV500). The shaker forced only $1 / 8^{\text {th }}$ part of the dome on a grid of 18 points, which was extended to a full grid of $15 \times 15$ points, using symmetry considerations. Thus a matrix of mobility transfer functions $\mathbf{H}$ of size $N_{r} \times N_{e}=225 \times 225$ was obtained for each frequency considered. The mobility transfer functions were measured in a frequency range up to $1000 \mathrm{~Hz}$, with a frequency resolution of 0.625 $\mathrm{Hz}$.

With $N_{e}=N_{r}=225, \sqrt{S / N_{e}}=\sqrt{S / N_{r}}=0.2 \mathrm{~m}$, it can be concluded that the discretization requirements related to the acoustic wavelength, as described in Section 2.2, are met up to a frequency of about $560 \mathrm{~Hz}$ ( $n=3$ sampling points per acoustic wavelength). Using the estimates that are given in Table 1 , requirement related to the structural wavelength $\lambda_{s}$ is fulfilled up to a frequency well above $330 \mathrm{~Hz}$, probably even higher.

\subsection{Operational deflection shapes and resonance frequencies}

A typical set of mobility transfer functions $\mathbf{H}$, as measured at one shaker excitation point, is shown in Fig. 8. The global coherence, as defined in [33], is shown as well. The global coherence is the ratio between the averaged coherent output power spectrum and the averaged output power spectrum of a SIMO (Single Input Multiple Output) system. It represents the part of the global output energy that is linearly related to the source. It is used here to avoid the superposition of several coherence curves that would be difficult to interpret.

From the measured matrix of mobility transfer functions $\mathbf{H}$, the operational deflection shapes were 


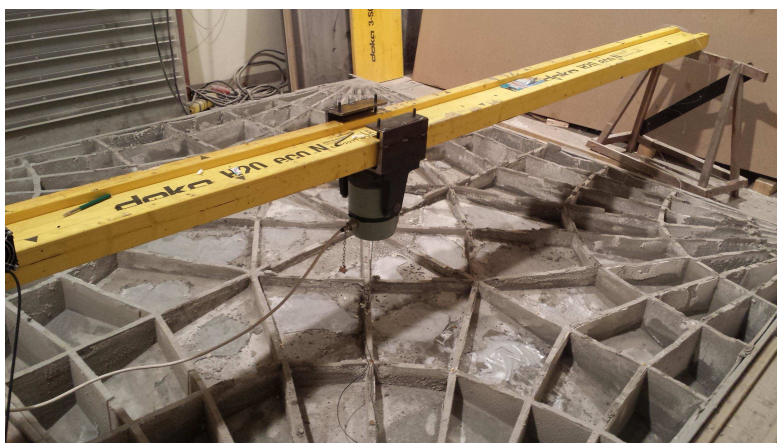

(a)

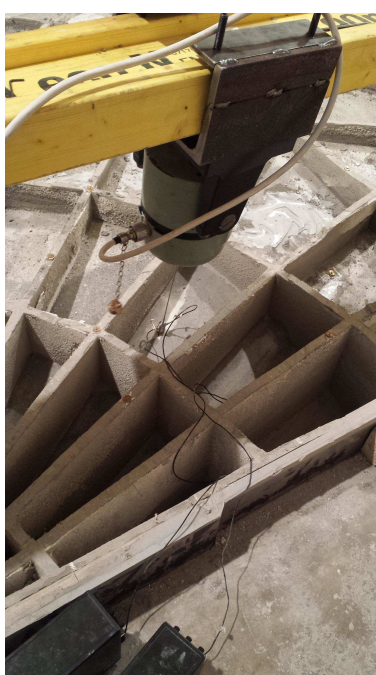

(b)



(c)
Figure 5: Funicular shell shaker setup, at the top of the funicular shell. Shaker excitation shown for some of the tested positions: a) on a rib near the center of the shell; b) in the bays between ribs; c) on a rib near the corner of the shell.

constructed. For six resonance frequencies of the funicular shell the operational deflection shapes are shown in Fig. 9. These shapes will closely resemble the eigenmodes of the funicular shell, as it are resonant operational deflection shapes.

\subsection{Measurement of the sound reduc- tion index according to the stan- dard ISO 10140-2:2010}

The measurement of the sound reduction index is prescribed in ISO 10140-2:2010 [9]. The methodology measures the sound pressure level in the source $\left(L_{p 1}\right)$ and receiving room $\left(L_{p 2}\right)$, averaged over a sufficient number of positions. The measurements were performed at eight fixed microphone positions (the standard ISO 10140-2:2010 requires at least five).

In order to account for the reverberation of the sound in the receiving room, and the size of the test opening, a correction term $10(\log S / A)$ needs to be added to the $\left(L_{p 1}-L_{p 2}\right)$ level difference, to obtain

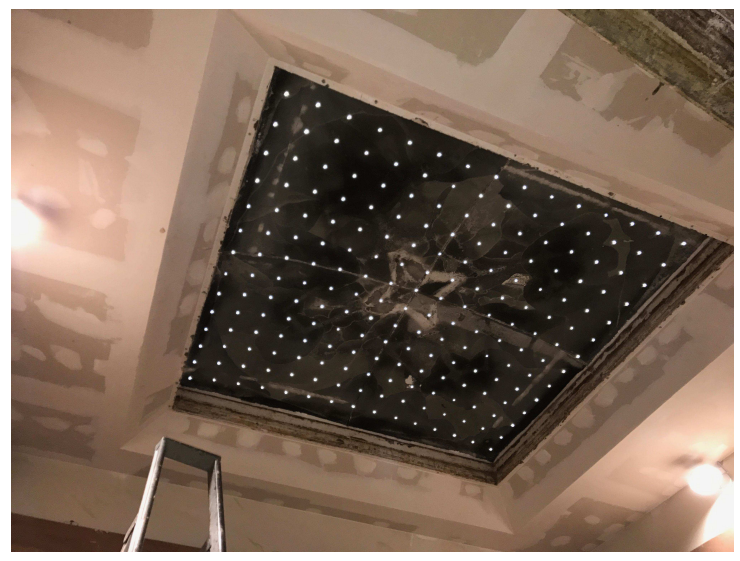

Figure 6: Funicular shell mounted in the opening of the transmission loss facility, with retro reflecting stickers glued on the dome, at the bottom.

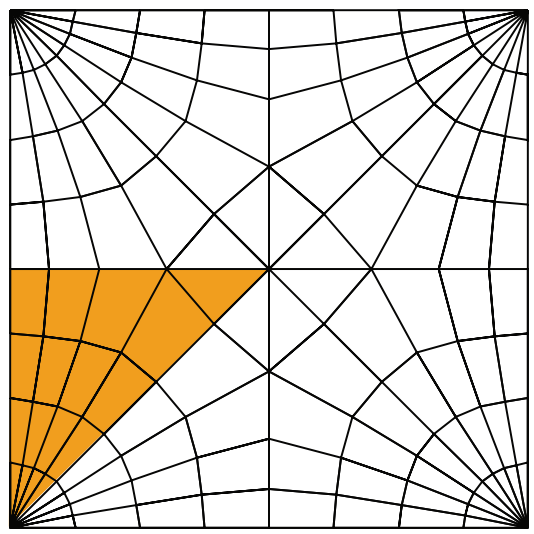

Figure 7: Exploiting symmetry of the funicular shell.

the sound reduction index $R$ :

$$
R=L_{p 1}-L_{p 2}+10 \log \left(\frac{S}{A}\right)
$$

where $S$ is the area of the free test opening in which the element is mounted, and $A$ the equivalent sound absorption area in the receiving room. The equivalent sound absorption area $A$ follows from the reverberation time measurements in the receiving room.

The uncertainty in the measured sound reduction index can be determined by considering the standard deviation of the sound pressure levels in the source and receiving room from position to position, $\sigma_{L_{p 1}}$ and $\sigma_{L_{p 2}}$, respectively, as well as the standard deviation of the equivalent sound absorption in the receiving room resulting from the reverberation time standard deviation[34]. Given that the different contributions to the total uncertainty on $R$ are independent, the combined uncertainty can then be estimated as $\sigma_{R}=\sqrt{\sigma_{L_{p 1}}^{2}+\sigma_{L_{p 2}}^{2}+\sigma_{10 \log (A)}^{2}}$. In case of repeated measurements on $N$ (eight) positions, it can be expected that the error on the mean reduces to $\sigma_{R, \bar{x}}=\sigma_{R} / \sqrt{N}$. 
Preprint submitted to Journal of Sound and Vibration, July 5, 2018, N.B. Roozen et al., p. 8

Table 1: Measured resonance frequencies and predicted eigenfrequencies of the funicular shell.

\begin{tabular}{|l|l|l|l|l|}
\hline $\begin{array}{l}\text { mode (letters referring to } \\
\text { Fig. 8a and to sub-figures } \\
\text { in Figs. 9 and 13. }\end{array}$ & $\begin{array}{l}\text { experimental } \\
\text { resonance } \\
\text { frequency }\end{array}$ & $\begin{array}{l}\text { numerical } \\
\text { eigen- } \\
\text { frequency }\end{array}$ & difference & $\begin{array}{l}\text { structural } \\
\text { wavelength }\end{array}$ \\
\hline a & $70 \mathrm{~Hz}$ & $71 \mathrm{~Hz}$ & $1 \mathrm{~Hz}, 1.4 \%$ & $\lambda_{s}>3 \mathrm{~m}$ \\
b & $107 \mathrm{~Hz}$ & $106 \mathrm{~Hz}$ & $1 \mathrm{~Hz}, 0.9 \%$ & $\lambda_{s} \approx 2.5 \mathrm{~m}$ \\
c & $155 \mathrm{~Hz}$ & $146 \mathrm{~Hz}$ & $9 \mathrm{~Hz}, 5.8 \%$ & $\lambda_{s} \approx 2.1 \mathrm{~m}$ \\
d & $178 \mathrm{~Hz}$ & $161 \mathrm{~Hz}$ & $17 \mathrm{~Hz}, 9.5 \%$ & $\lambda_{s} \approx 1.5 \mathrm{~m}$ \\
e & $286 \mathrm{~Hz}$ & $268 \mathrm{~Hz}$ & $18 \mathrm{~Hz}, 6.3 \%$ & $\lambda_{s} \approx 1.2 \mathrm{~m}$ \\
f & $327 \mathrm{~Hz}$ & $296 \mathrm{~Hz}$ & $31 \mathrm{~Hz}, 9.5 \%$ & $\lambda_{s} \approx 1.2 \mathrm{~m}$ \\
\hline
\end{tabular}

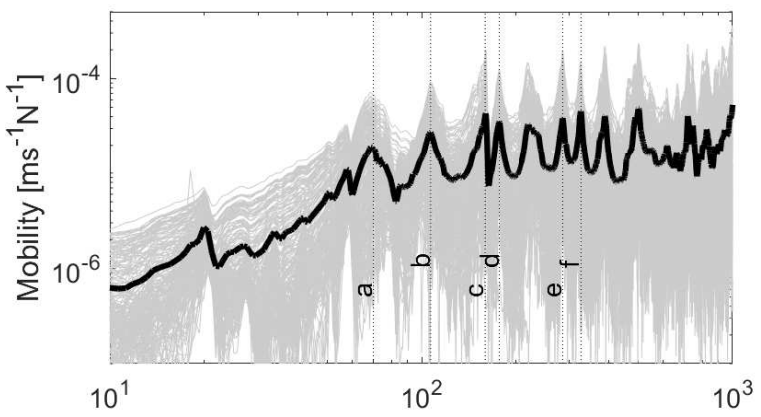

(a)

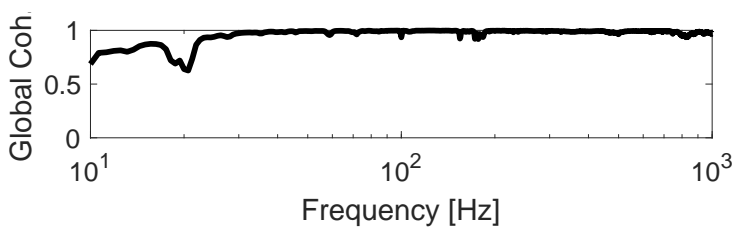

(b)

Figure 8: Measured mobilities of the funicular shell: a) Transfer functions $\mathbf{H}$ as measured at one shaker excitation point (gray curves) and the spatially mean mobility transfer function (black thick solid curve). The letters a-f refer to the rows in Table 1; b) Global coherence as defined in [33].

Following this approach, the sound reduction index $R$ of the funicular shell was estimated as shown in Fig. 10. The mean $R$ incremented and decremented with the standard deviation $\sigma_{R}$, as well as incremented and decremented by the standard error $\sigma_{R, \bar{x}}^{2}$ are shown in this figure as well.

In Fig. 11 the individual standard deviations are shown. From this figure it is clear that the largest standard deviations are caused by the variation of the sound pressure levels in the source and receiving rooms, $\sigma_{L_{p 1}}$ and $\sigma_{L_{p 2}}$. The standard deviation of the correction term that accounts for the reverberation time of the receiving room, $\sigma_{10} \log (A)$, is smaller.

In Fig. 10 and Fig. 11 it can be seen that the uncertainty in the sound reduction index $R$ increases with decreasing frequency, being as large as almost 8 $\mathrm{dB}$ for some frequencies below $100 \mathrm{~Hz}$.

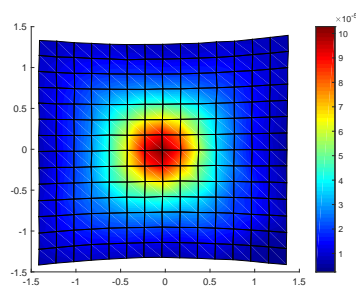

(a) $70 \mathrm{~Hz}$

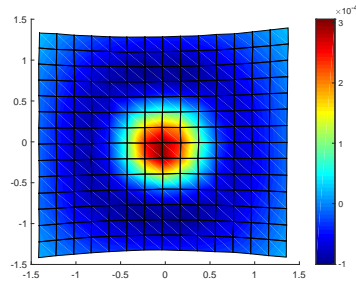

(c) $155 \mathrm{~Hz}$

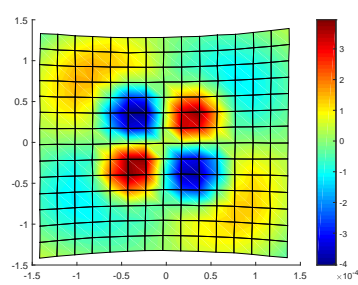

(e) $286 \mathrm{~Hz}$

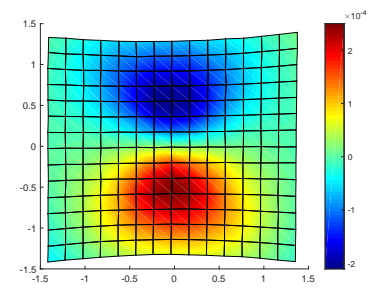

(b) $107 \mathrm{~Hz}$



(d) $178 \mathrm{~Hz}$

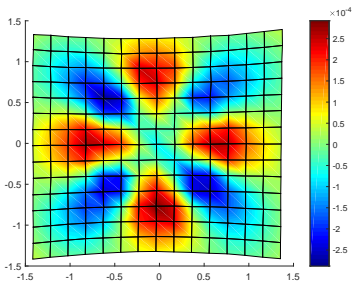

(f) $327 \mathrm{~Hz}$
Figure 9: Measured funicular shell operational deflection shapes for different frequencies: a) $70 \mathrm{~Hz}$; b) 107 Hz; c) $155 \mathrm{~Hz}$ d) $178 \mathrm{~Hz}$; ) $286 \mathrm{~Hz}$ f) $327 \mathrm{~Hz}$.

\subsection{Mobility based estimation of the sound reduction index}

The mobility-based estimate of the sound reduction index, following the theory as described in Section 2, is shown in Fig. 12, together with the ISO10140-2 results. The figure shows that the mobility-based estimate of the sound reduction index $R$ stays within the uncertainty range of the ISO10140-2 result. Towards the higher frequencies, up to a frequency of about 500 $\mathrm{Hz}$, the mobility-based estimate of the sound reduction index coincides well with the ISO result, thus vali- 


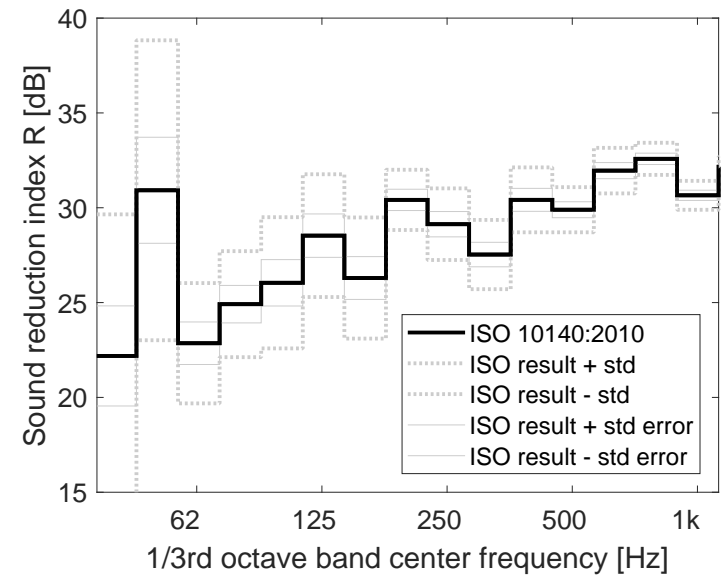

Figure 10: Sound reduction index $R$ of the funicular shell, as measured according to ISO 10104-2:2010, from 50 to $1000 \mathrm{~Hz}$. The interval between the dotted and full gray lines indicate the estimated standard deviation between measurements on different microphone positions and the standard error on their mean respectively.

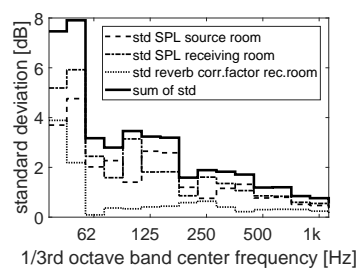

(a)

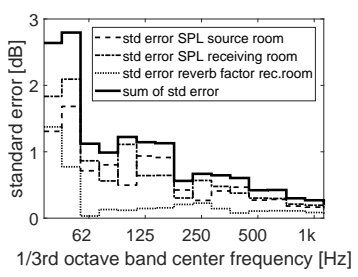

(b)
Figure 11: Standard deviation (a) and standard error (b) on the sound pressure level measured on different microphone positions in the source (dashed line) and receiving (dash-dotted line) room, on the correction term (dotted line) and square root of the quadratic sum of the standard deviations (thick solid line).

dating again the proposed procedure. In the lower frequency range, large variations of the sound reduction index $R$ can be observed for the microphone based standardized method, whilst the mobility based approach shows less strong variations as function of frequency. The larger variations that are shown by the standardized approach are believed to be caused by the breakdown of the diffuse field assumption at low frequencies. As such, the proposed method can be considered complementary to the standardized measurement approach.

The proposed mobility-based estimate of the sound reduction index requires a larger measurement effort as compared to the standardized ISO10140-2 measurement approach. The measurement of the matrix of mobilities in this particular case of the funicular shell required about 1 full working day, whereas the ISO measurement approach required a measurement

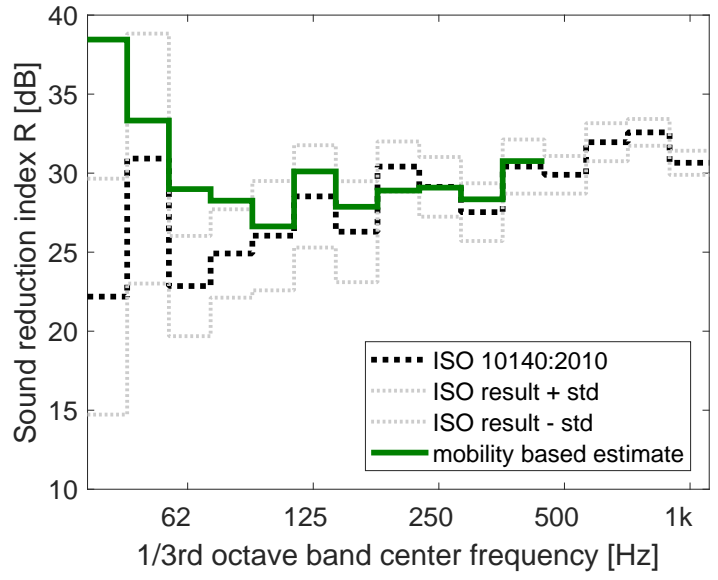

(a)

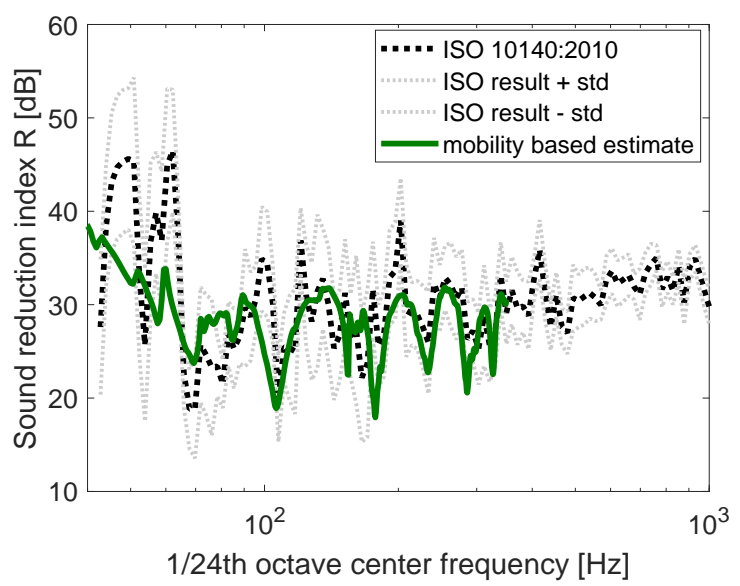

(b)

Figure 12: Sound reduction index $R$ of the funicular shell, measurements according to ISO 10140-2:2010 versus mobility-based estimate. a) Results presented in $1 / 3^{\text {rd }}$-octave bands, b) results presented in $1 / 24^{\text {th }}$ octave bands for the ISO method, and in FFT narrow bands for the mobility based result.

time of about 1 hour. The larger measurement effort of the mobility-based approach is mainly due to the sequential measurement of each mobility transfer function. Non-sequential measurement approaches would reduce the measurement effort.

\subsection{Finite element model, comparison with mobility-based results and convergence considerations}

For the complex geometry of the funicular shell, it is not possible to obtain an analytical solution. Therefore a numerical finite element method (FEM) model was built to validate the mobility-based estimate of the sound reduction index. The model consisted of 76,615 nodes and 38,860 shell elements.

The computed eigenmodes that were obtained with 


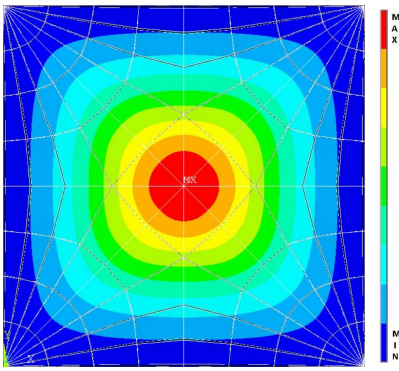

(a) $71 \mathrm{~Hz}$

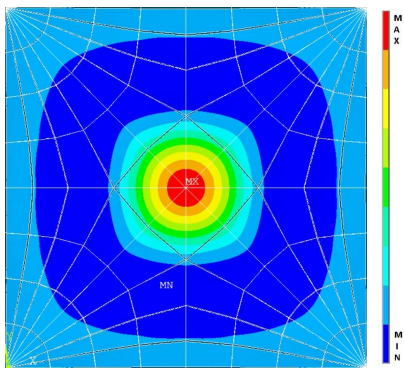

(c) $146 \mathrm{~Hz}$

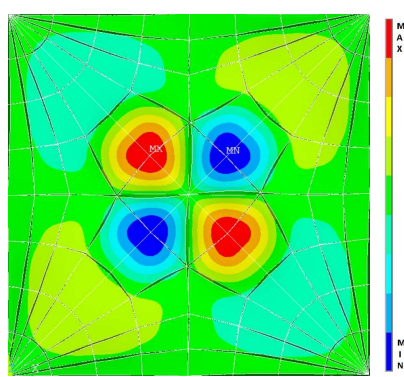

(e) $268 \mathrm{~Hz}$

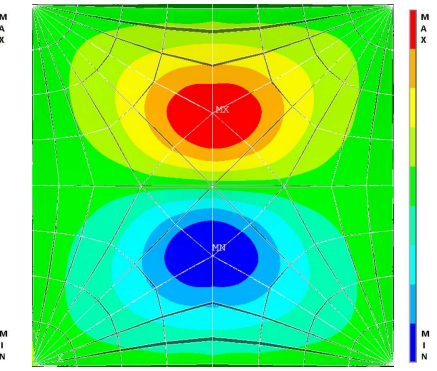

(b) $106 \mathrm{~Hz}$

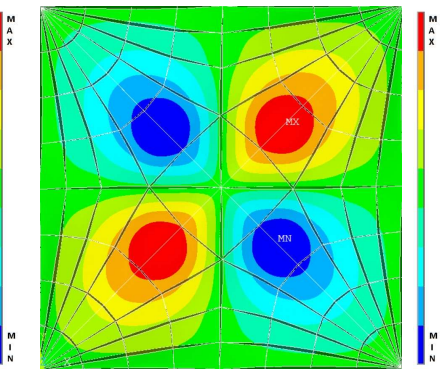

(d) $161 \mathrm{~Hz}$

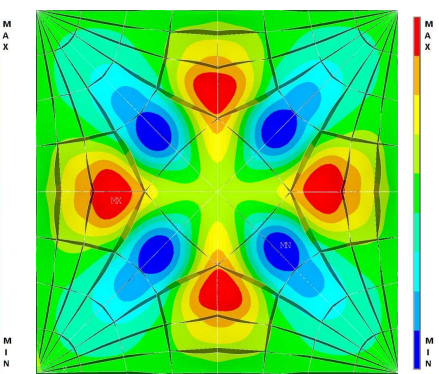

(f) $296 \mathrm{~Hz}$
Figure 13: FE-predicted funicular shell eigenmodes with eigenfrequencies: a) $71 \mathrm{~Hz}$; b) $106 \mathrm{~Hz}$; c) 146 $\mathrm{Hz}$; d) $161 \mathrm{~Hz}$; e) $268 \mathrm{~Hz}$ f) $296 \mathrm{~Hz}$.

the numerical FEM model are presented in Figure 13, showing a reasonable correspondence with the experimentally obtained operational deflection shapes at the resonance frequencies (see Fig.9 and Table 1).

Using the finite element model, mobility transfer functions were computed for an excitation and response grid of $15 \times 15$ points, similar to the grids used for the experiments on the funicular shell. The sound reduction index was computed on the basis of these mobilities as detailed in Section 2. To investigate the convergence behavior of the mobility-based estimate of the sound reduction index, an excitation and response grid of $30 \times 30$ points (resulting in a matrix of mobility transfer functions $\mathbf{H}$ with a dimension of $N_{r} \times N_{e}=900 \times 900$, for each frequency considered) was considered as well. The sound reduction index as computed with the FEM-model, using both excitation and response grids, is shown in Fig. 14. It shows that up to a frequency of about $200 \mathrm{~Hz}$ the excitation and response grid of $15 \times 15$ points does not deviate from

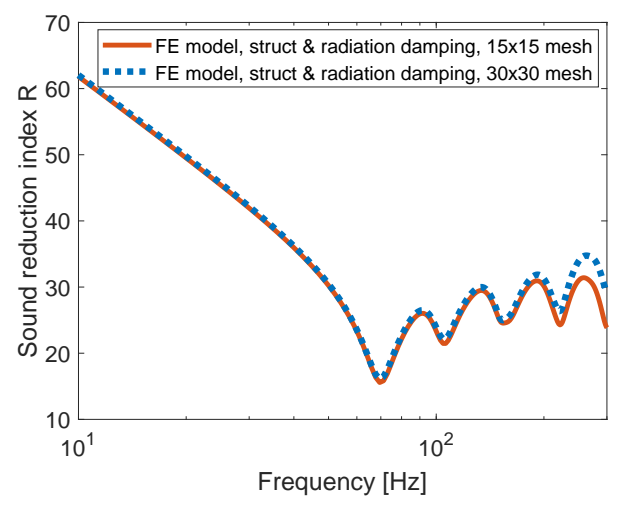

Figure 14: Sound reduction index $R$ of the funicular shell, using FE-predicted transfer functions, with a 15x15 mesh (solid brown curve) and a 30x30 mesh (dashed blue curve). Results are presented in FFT narrow bands.

the results obtained with the $30 \times 30$ points grid, indicating that the $15 \times 15$ points grid is sufficiently fine for the estimation of the sound reduction index of the funicular shell up to $200 \mathrm{~Hz}$.

In the finite element model the in-vacuo structure is being considered, which implies that the effect of radiation damping is not taken into account. Having computed a matrix of transfer functions $\mathbf{H}$ "invacuo", the radiation damping can be taken into account afterwards as described in Appendix B. Figure 15 compares the mobility-based estimate of the sound reduction index, based on the measured matrix of mobilities, and the computed mobilities of the finite element model. The latter is shown with and without the effect of radiation damping.

The results shown in Fig. 15 shows a reasonable correspondence between the $R$ obtained from the finite element model and from the mobility measurements. This again validates the mobility based approach. The sound reduction index is shown for frequencies down to $10 \mathrm{~Hz}$. The first resonance frequency of the funicular shell is about $70 \mathrm{~Hz}$, causing the sound insulation below that frequency to be stiffness controlled, which is characterized by an $R$ with a $40 \mathrm{~dB} / \mathrm{dec}$ slope. Both the results obtained by the FE-model and the mobility based measurement results show a stiffness controlled behavior for frequencies below $70 \mathrm{~Hz}$. 


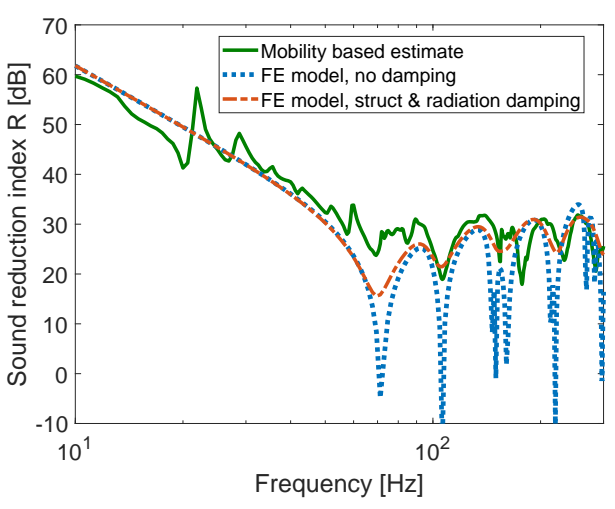

Figure 15: Sound reduction index $R$ of the funicular shell, mobility-based (green solid curve) estimate versus FE-model result for undamped case (blue doted curve) and for the case including structural damping (5\% critical) and radiation damping (dash-dotted brown curve). Results presented in FFT narrow bands.

\section{Conclusions}

A new hybrid approach was proposed to measure the sound reduction index of a partition without the adverse effects of the acoustic properties of the source and receiving rooms at frequencies below the Schroeder frequency, yielding the true sound reduction index of the partition. In this new approach mobility measurements are combined with a numerical procedure.

The approach was shown to be consistent with the standardized ISO10140-2:2010 measurement approach for frequencies just above the Schroeder frequency. The ISO10140-2:2010 is based on the diffuse field assumption. Below the Schroeder frequency significant differences between the new approach and ISO10140-2:2010 were observed, which are believed to be caused by the breakdown of the diffuse field assumption at low frequencies.

The new approach is especially suited for the frequency range below the Schroeder frequency, and is as such complementary to the standardized measurement approach. It is expected that the new approach is also suitable for in-situ measurements. Furthermore, the method is unaffected by the presence of possible flanking paths.

During the experiments a matrix of mobilities was measured along a grid of excitation and response points, sequentially in time. Consequently the proposed method required a larger measurement effort as compared to the standardized ISO10140-2 measurement approach. Emerging measurement technologies, such as automated scanning laser Doppler measurement techniques, now already allow for accurate and fast mobility measurements that are required for this approach. With the advance of full-field optical measurement techniques like digital image correlation techniques [35] and full-field digital holographic inter- ferometry techniques [36] the measurement effort can be further reduced. Alternatively, a grid of accelerometers can be used to capture the vibrational response of the partition in one single measurement session per excitation point. These full-field measurement techniques as well as the use of a grid of accelerometers would also allow to excite the partition by means of an instrumented hammer, which will make the measurement more easy to perform as compared to a shaker excitation. Next to the larger measurement effort, also a larger numerical effort is required to compute the cross spectral matrix of structural responses and to compute the radiated sound power by means of the Rayleigh integral. This numerical part can be well described in standardized procedures and be performed at every measurement facility.

Despite the larger experimental-numerical effort as compared to the standardized approach, the authors believe it is a viable and realistic alternative to standardized approaches for frequencies below the Schroeder frequency. It is expected that the approach presented in this manuscript will motivate a discussion on the normalization of measurement procedures for the lower frequency range.

\section{Acknowledgments}

This work was carried out in the framework of H2020MSCA-RISE-2015 No. 690970 "Papabuild", and was partly funded by the Post-doctoral seed funding grant from the Partnership Council for Sustainable Construction given by the ETH Foundation.

The following people from KU Leuven are acknowledged: Stephan Solinas, Bernd Salaets, Daniel Otero and Pengchao Wang for their help in the construction of the funicular shell; Willy Bruyninckx for performing the measurements according to the standard; Ludovic Labelle for his help in setting up part of the measurement equipment; Luca Sangiuliano and Noé Geraldo Rocha de Melo Filho for their help with the scanning laser Doppler vibrometer; Edwin Reynders for coordinating the funicular cell construction works.

\section{Appendices}

\section{A Computation of the radia- tion impedance matrix}

Consider a baffled partition radiating into a semiinfinite acoustic domain, having a velocity field that is described by $v\left(\mathbf{x}_{e}, \omega\right)$. The pressure on the radiating surface can be computed by means of the Rayleigh integral $[15,16,26]$, which reads:

$$
p\left(\mathbf{x}_{r}, \omega\right)=\frac{\mathrm{i} \omega \rho}{2 \pi} \iint_{S} v_{n}\left(\mathbf{x}_{e}, \omega\right) \frac{e^{-\mathrm{i} k r}}{r} \mathrm{~d} S_{r}
$$


where $r$ is the distance between the points in vector $\mathbf{x}_{e}$ and vector $\mathbf{x}_{r}, k$ is the wavenumber, $\rho$ the density and $\omega$ the radial frequency. Note that the above integral has a singularity for $r \rightarrow 0$.

Equation 17 can be written in matrix form as

$$
\mathbf{p}=\mathbf{Z} \mathbf{v}
$$

where the matrix $\mathbf{Z}$ can be computed as follows [27], taking into account the singularity at $r \rightarrow 0$ :

$$
\mathbf{Z}=\rho c\left[\begin{array}{ccc}
d & -\frac{\mathrm{i} k S}{2 \pi} \frac{e^{\mathrm{i} k r_{i j}}}{r_{i j}} & \cdots \\
-\frac{\mathrm{i} k S}{2 \pi} \frac{e^{\mathrm{i} k r_{j i}}}{r_{j i}} & d & \cdots \\
\vdots & \vdots & \ddots
\end{array}\right]
$$

where

$$
d=\frac{1}{2}\left(k \sqrt{\frac{S}{\pi}}\right)^{2}-\mathrm{i} \frac{8}{3 \pi}\left(k \sqrt{\frac{S}{\pi}}\right)
$$

\section{B An analytical model to pre- dict the airborne sound re- duction of a simply sup- ported plate under a diffuse acoustic excitation, including radiation damping effects}

The analytic model for the simply supported flexural rectangular plate is considered here. The displacement $w$ of the plate excited by a unitary point force at the coordinates $\left(x_{e}, y_{e}\right)$, for the angular frequency $\omega$, can be described by a modal expansion [37]:

$$
\begin{gathered}
=\frac{4}{D L_{x} L_{y}} \sum_{i, j \geq 1} \frac{\sin \left(k_{i} x_{e}\right) \sin \left(k_{j} y_{e}\right) \sin \left(k_{i} x\right) \sin \left(k_{j} y\right)}{\left(k_{i}^{2}+k_{j}^{2}\right)^{2}-k_{F}^{4}} \\
\text { with } k_{i}=\frac{i \pi}{L x}, k_{j}=\frac{j \pi}{L y}, k_{F}=\left(\omega^{2} \frac{\rho h}{D}\right)^{1 / 4}
\end{gathered}
$$

where $L_{x}$ and $L_{y}$ are the plate's dimensions and $D$ is the dynamic flexural rigidity defined by $D=$ $\frac{E(1+\mathrm{i} \eta) h^{3}}{12\left(1-\nu^{2}\right)}$ where $h$ is the plate thickness, $E$ is Young's modulus, $\eta$ is the loss factor, $\rho$ is the density and $\nu$ is Poisson's ratio. From Eq. 21 the matrix of transfer functions $\mathbf{H}$, as defined by Eq. 2, can be constructed. However, this matrix of transfer functions relates the vector of velocities $\mathbf{v}$ to the vector of exciting forces $\mathbf{f}$ for the the in-vacuo structure. Below a method is presented to take the radiation damping into account as a simple modification of the matrix of transfer functions $\mathbf{H}$.

Recalling Eq. 2, we have

$$
\mathbf{v}=\mathbf{H f}
$$

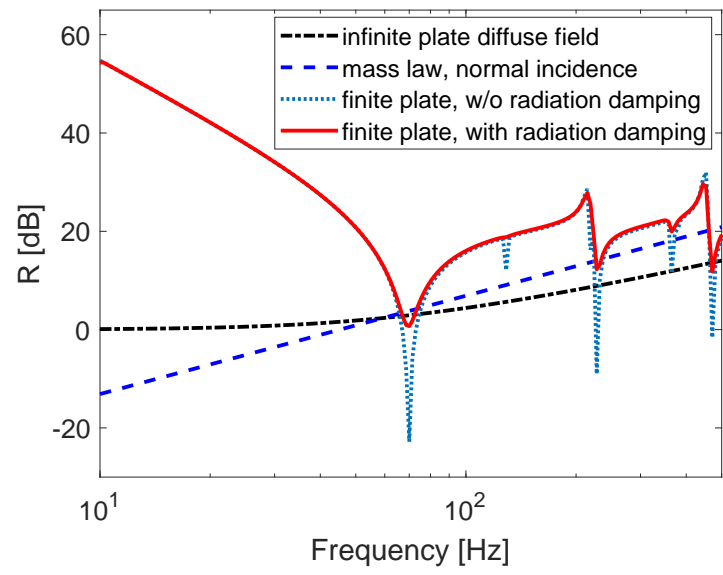

Figure 16: Diffuse field sound reduction index $R$ of an aluminum plate, $L_{x}=0.35 \mathrm{~m}, L_{y}=0.22 \mathrm{~m}$, plate thickness $h=0.001 \mathrm{~m}$, computed from the theoretical mobility data of a simply supported plate (Eq. 21).

The radiated pressure on the source side and on the receiving side, can be accounted for by means of an additional load on the structure, resulting in the modified relationship

$$
\mathbf{v}=\mathbf{H}\left(\mathbf{f}-2 \mathbf{d} \mathbf{S} \mathbf{p}_{\mathbf{r}}\right)
$$

where $\mathbf{p}_{r}$ is the radiated pressure field.

Using the radiation impedance matrix for an infinite acoustic half space, $\mathbf{Z}[16,26,27]$ (see Appendix A), which links the parietal pressure on the source side and on the receiving side radiated to the vibrating structure having a velocity field $\mathbf{v}$, the above relationship can be written as

$$
\mathbf{v}=\mathbf{H}(\mathbf{f}-2 \mathbf{d} \mathbf{S} \mathbf{Z} \mathbf{v})
$$

and thus

$$
(\mathbf{I}+2 \mathbf{H} \mathbf{d S} \mathbf{Z}) \mathbf{v}=\mathbf{H f}
$$

where $\mathbf{I}$ is the identity matrix, yielding

$$
\mathbf{v}=(\mathbf{I}+2 \mathbf{H} \mathbf{d} \mathbf{S} \mathbf{Z})^{-1} \mathbf{H} \mathbf{f}=\mathbf{H}_{\bmod } \mathbf{f}(\omega)
$$

where $\mathbf{H}_{m o d}$ is the transfer function that takes into account the radiation load, assuming two infinite half spaces on either side of the structure, and thus also accounts for the radiation damping of the structure.

To validate the procedure to compute the diffuse field sound reduction index $R$ from mobility data, a single aluminum plate as described by Sgard (JASA 2000) [32], with dimensions $L_{x}=0.35 \mathrm{~m}, L_{y}=0.22 \mathrm{~m}$, $h=0.001 \mathrm{~m}, E=71 \mathrm{GPa}, \nu=0.33, \rho=2814 \mathrm{~kg} / \mathrm{m}^{3}$ and $\eta=0.001$, was considered. A discretization mesh was used of $N_{e}=N_{r}=20 \times 20=400$ points for both the excitation field and response field. Using the modal expansion given above, including eigenfrequencies up to twice the frequency range of interest, the response of the plate due to a diffuse acoustic excitation was 
computed using equations 1 and 5 . Subsequent use of the equations 8 and 11, yielded the sound reduction index $R$ according to the theoretical model. Fig. 16 shows the computed theoretical sound reduction index $R$, for the case structural damping is considered only, $\eta=1 \%$, and for the case the radiation damping is considered as well, using the modified mobility transfer matrix $\mathbf{H}_{\text {mod }}$ given in Eq. 26.

The results compare well with the results published by Sgard [32] (figure 2 on page 2868 of that publication) for the case that radiation damping is not taken into account. This validates the procedure to compute the diffuse field sound reduction index $R$ from mobility data.

It is noteworthy to mention that the inclusion of radiation damping is essential to have predictions of the sound reduction index $R$ which are non-negative for all frequencies. From a physical point of view a negative sound reduction index $R$ is not possible. Only when the effects of radiation damping is taken into account, the energy balance is modeled correctly, making it impossible to have negative $R$-values. See also Section 3.2, Fig. 4 and Section 4.5, Fig. 15 for example calculations that illustrate this aspect.

\section{References}

[1] B. Berglund, P. Hassmen, R. Job, Sources and effects of low-frequency noise, J. Acoust. Soc. Am. 99 (5) (1996) 2985-3002.

[2] M. Rychtarikova, H. Muellner, V. Chmelik, N. B. Roozen, D. Urban, D. P. Garcia, C. Glorieux, Perceived Loudness of Neighbour Sounds Heard Through Heavy and Light-Weight Walls with Equal R-w + C50-5000, Acta Acust. united Ac. 102 (1) (2016) 58-66.

[3] J. Mahn, J. Pearse, The Uncertainty of the Proposed Single Number Ratings for Airborne Sound Insulation, Building Acoustics 19 (3) (2012) 145172 .

[4] W. Scholl, J. Lang, V. Wittstock, Rating of Sound Insulation at Present and in Future. The Revision of ISO 717, Acta Acust. united Ac. 97 (4) (2011) 686-698.

[5] ISO 717-1:2013, acoustics - rating of sound insulation in buildings and of building elements part 1: Airborne sound insulation.

[6] V. Hongisto, J. Keranen, M. Kylliainen, J. Mahn, Reproducibility of the Present and the Proposed Single-Number Quantities of Airborne Sound Insulation, Acta Acust. united Ac. 98 (5) (2012) 811-819.
[7] C. Scrosati, F. Scamoni, M. Bassanino, M. Mussin, G. Zambon, Uncertainty analysis by a Round Robin Test of field measurements of sound insulation in buildings: Single numbers and low frequency bands evaluation - Airborne sound insulation, Noise Contr. Eng. J. 61 (3) (2013) 291-306.

[8] M. Machimbarrena, C. R. A. Monteiro, S. Pedersoli, R. Johansson, S. Smith, Uncertainty determination of in situ airborne sound insulation measurements, Appl. Acoust. 89 (2015) 199-210.

[9] ISO 10140-2:2010, acoustics - laboratory measurement of sound insulation of building elements - part 2: Measurement of airborne sound insulation.

[10] A. Tadeu, J. Antonio, D. Mateus, Sound insulation provided by single and double panel walls - a comparison of analytical solutions versus experimental results, Appl. Acoust. 65 (1) (2004) $15-29$.

URL http://www.sciencedirect.com/ science/article/pii/S0003682X03001130

[11] C. Hopkins, P. Turner, Field measurement of airborne sound insulation between rooms with non-diffuse sound fields at low frequencies, Appl. Acoust. 66 (12) (2005) 1339-1382.

[12] E. de A. C. Duarte, A. Moorhouse, E. B. Viveiros, Indirect measurement of acoustic power into a small room at low frequencies, Appl. Acoust. 73 (3) (2012) 248-255.

[13] T. Bravo, S. Elliott, Variability of low frequency sound transmission measurements, J. Acoust. Soc. Am. 115 (6) (2004) 2986-2997.

[14] T. Bravo, C. Maury, Enhancing low frequency sound transmission measurements using a synthesis method, J. Acoust. Soc. Am. 122 (2) (2007) 869-880.

[15] N. B. Roozen, L. Labelle, M. Rychtarikova, C. Glorieux, Determining radiated sound power of building structures by means of laser Doppler vibrometry, J. Sound Vib. 346 (2015) 81-99.

[16] G. Koopmann, H. Benner, Method for computing the sound power of machines based on the Helmholtz integral, J. Acoust. Soc. Am. 71 (1) (1982) 78-89.

[17] N. B. Roozen, Q. Leclère, D. Urban, L. Kritly, C. Glorieux, Assessment of the sound reduction index of building elements by near field excitation through an array of loudspeakers and structural response measurements by laser Doppler vibrometry, Appl. Acoust. 140 (2018) 225-235. 
[18] P. Gardonio, M. Brennan, On the origins and development of mobility and impedance methods in structural dynamics, J. Sound Vib. 249 (3) (2002) 557-573.

[19] P. Gardonio, M.J. Brennan, Mobility and impedance methods in structural dynamics, in: F. Fahy, J. Walker (Eds.), Advanced Applications in Acoustics, Noise and Vibration, London, 2004, pp. 389-447.

[20] E. A. Piana, A method for determining the sound reduction index of precast panels based on point mobility measurements, Appl. Acoust. 110 (2016) 72-80.

[21] C. Marchetto, L. Maxit, O. Robin, A. Berry, Vibroacoustic response of panels under diffuse acoustic field excitation from sensitivity functions and reciprocity principles, J. Acoust. Soc. Am. 141 (6) (2017) 4508-4521.

[22] J.-D. Chazot, J.-L. Guyader, Prediction of transmission loss of double panels with a patchmobility method, J. Acoust. Soc. Am. 121 (1) (2007) 267-278.

[23] A. Piersol, Use of coherence and phase data between 2 receivers in evaluation of noise environments, J. Sound Vib. 56 (2) (1978) 215-228.

[24] H. Nelisse, J. Nicolas, Characterization of a diffuse field in a reverberant room, J. Acoust. Soc. Am. 101 (6) (1997) 3517-3524.

[25] F. Jacobsen, T. Roisin, The coherence of reverberant sound fields, J. Acoust. Soc. Am. 108 (1) (2000) 204-210.

[26] F. Fahy, Sound and Structural Vibration: Radiation, Transmission and Response., Academic Press, London, 1985.

[27] M. Bai, M. Tsao, Estimation of sound power of baffled planar sources using radiation matrices, J. Acoust. Soc. Am. $112(3,1)$ (2002) 876-883.

[28] F. Jacobsen, The sound field in a reverberation room, Tech. rep., Note no 31261, Technical University of Denmark (2008).

[29] A. J. Jerri, The shannon sampling theorem - its various extensions and applications - a tutorial review, Proceedings of the IEEE 65 (11) (1977) 1565-1596. URL <GotoISI> : //WOS : A1977EB77800004

[30] G. Pavic, Air-borne sound source characterization by patch impedance coupling approach, J. Sound Vib. 329 (23) (2010) 4907-4921.
[31] N. B. Roozen, Q. Leclere, K. Ege, Y. Gerges, Estimation of plate material properties by means of a complex wavenumber fit using hankel's functions and the image source method, J. Sound Vib. 390 (2017) 257-271.

URL ://WOS :000392162600014

[32] F. Sgard, N. Atalla, J. Nicolas, A numerical model for the low frequency diffuse field sound transmission loss of double-wall sound barriers with elastic porous linings, J. Acoust. Soc. Am. 108 (6) (2000) 2865-2872.

[33] N. B. Roozen, Q. Leclere, M. Rychtarikova, C. Glorieux, A global error estimator for the uncertainty of a multi-channel spectral analysis, Appl. Acoust. 87 (2015) 57-63.

[34] V. Wittstock, On the uncertainty of singlenumber quantities for rating airborne sound insulation, Acta Acust. united Ac. 93 (3) (2007) $375-386$.

[35] F. Chen, X. Chen, X. Xie, X. Feng, L. Yang, Fullfield 3D measurement using multi-camera digital image correlation system, Opt. Laser Eng. 51 (9) (2013) 1044-1052.

[36] D. De Greef, J. Soons, J. J. J. Dirckx, Digital Stroboscopic Holography Setup for Deformation Measurement at Both Quasi-Static and Acoustic Frequencies, Int. J. Optomechatroni $8(4, \mathrm{SI})$ (2014) 275-291.

[37] Q. Leclere, C. Pezerat, Vibration source identification using corrected finite difference schemes, J. Sound Vib. 331 (6) (2012) 1366-1377. 